\title{
Preface to Volume 2
}

This second volume of Helmut Wielandt's Mathematical Works contains his contributions to matrix theory and analysis.

In about two dozen papers of moderate length, Wielandt has deeply influenced matrix theory and linear algebra, and those parts of analysis and numerical mathematics that he investigated. Wielandt is a highly creative mathematician who had a vision, unusual for his time, of the proper balance in mathematics between the abstract and general on the one hand and the more concrete and specific on the other, as is demonstrated by his extraordinarily prescient acceptance speech to the Heidelberg Academy in 1961/62 reprinted in these volumes. Add to this the legendary sparseness and beauty of his style, and perhaps one obtains some explanation of the continuing importance of Wielandt's work.

A word needs to be said about the nature of the commentaries in this volume. I asked contributors to choose the paper they wished to discuss, and this has resulted in several commentaries being received on some papers and none on others. Contributors were also asked to remark on recent developments of the topics under discussion in order to make their observations useful to researchers of today. Most contributors complied, and thus some of the articles are expository papers that go well beyond what is usually understood by a commentary. A few articles are not even specifically tied a publication of Wielandt's. The reader is referred to the contents page for details. It is remarkable how little overlap there is in commentaries on the same paper and in articles on related topics, which again attests to scope of the development of Wielandt's ideas.

The commentaries appear after the corresponding publications, except that Olga Taussky's many contributions appear in one article by her expressed wish. My thanks are due to all contributors (whose names are listed on a separate page), and to my students, Ahmad Muchlis and Judith McDonald, who helped in organizing the commentaries. 